\title{
STUDY OF IDENTIFICATION OF TWO-PHASE FLOW PARAMETERS BY PRESSURE FLUCTUATION ANALYSIS
}

\author{
Ondrej Burian*, Vaclav Dostal, LadislaV Vesely
}

\author{
Faculty of Mechanical Engineering, Czech Technical University in Prague, Technická 4, Praha 6, Czech Republic \\ * corresponding author: ondrej.burian@fs.cvut.cz
}

\begin{abstract}
This paper deals with an identification of a parameters of simple a pool boiling in a vertical rectangular channel by analysis of pressure fluctuation. A small experimental facility about $9 \mathrm{~kW}$ power, which was used for simulation of apool boiling phenomena and creation of steam-water volume in this work is introduced. A several pressure fluctuations measurements and differential pressure fluctuations measurements at various were carried out. Main changed parameters were a power of heaters and a hydraulics resistance of a channel internals. A measured pressure data were statistically analysed and compared with goal to find dependencies between parameters of a two-phase flow and statistical properties of pressure fluctuation. At the end of this paper are summarized final results and applicability of this method for parameters determination of two phase flow for pool boiling conditions at ambient pressure is discused.
\end{abstract}

KEYWORDS: two-phase flow, pressure fluctuation measurement, void fraction.

\section{INTRODUCTION}

Boiling of liquids is a high dynamic phenomena, which is characterized as a phase change of a liquid phase to a gaseous phase. The most common example of that is boiling of water to water steam, which occurs in many facilities in our life. It may exist in wide range of parameters and regiments, which includes pressure, temperature, heat flux, bulk temperature etc. The one of the key parameters is a void fraction which represents a share of steam fraction in overall volume. The knowledge of this parameter is key for identification other parameters of boiling and operational parameters of facilities which operate with boiling phenomena like steam boilers, boiling water reactors or steam generators.

One of a perspective method for identification a key parameters of boiling and two-phase flow phenomena, which is focused on analysis of measured differential pressure and its a time fluctuation, is described in this work. This method is based on fact that differential pressure between two measured locations in a two-phase mixture located in some distance at above is dependent on the void fraction. Void fraction in volumetric form is defined as a ratio of a steam fraction volume to overall a two-phase volume, see (1). The density of a two-phase mixture can be expressed by $(2)$, where $\rho_{l}$ is density of liquid fraction and $\rho_{g}$ is density of steam fraction.

$$
\begin{gathered}
\alpha=\frac{V_{g}}{V} \\
\rho_{m}=\rho_{l}(1-\langle\alpha\rangle)+\rho_{g}\langle\alpha\rangle
\end{gathered}
$$

Second assumption is that the value of this differential pressure is not stable in stable state, but it fluctuated around some mean value. This dependency is a result of influence of the void fraction on a hydrostatic pressure at measured locations - a measurement points. Hydrostatic pressure of a two-phase volume about some void fraction is value between hydrostatic pressure of a liquid volume and a steam volume, which decreases with increasing a void fraction. A pressure fluctuation is a result of a process of a bubble generation and its flow throw the measured section.

If we find dependency between void fraction and measured differential pressure, which is measured between two measurement points located at above in some distance, we can use it like fundamental of a method for determination of the void fraction. However more interesting may be an information about boiling and two-phase flow parameters which we can get from statistical analysis of fluctuation of measured differential pressure. Research of this method, identification advantages and disadvantages of this method is subject of this research work which will be introduced in this paper.

Due to a boiling and a two-phase flow are complicated phenomenas, is impossible to determinate an analytical solution with physical model of dependency between void fraction and differential pressure. They are complex phenomenas which are affected many external and internal influences. From that reasons as described at above was chose experimental way which should give empirical view of scope to this problem. Using differential pressure as a parameter is more useful than point pressure, because we do not know position of a level of a two-phase mixture up the measured points.

The beginning phase of this research project is focused on an experimental research at an ambient pressure conditions. The aim of this phase is a verification an initial assumptions, assessment a general values of 